\title{
Innovación e intercambio de conocimiento en la historia de la planificación: una mirada desde Europa ${ }^{1}$
}

Stephen Ward. Oxford Brookes University, Oxford, Reino Unido.

RESUMEN | Tradicionalmente la historia de la planificación se ha escrito en términos de innovaciones e innovadores: las "brillantes ideas" de la planificación y aquellos pioneros que las pensaron y que fueron capaces de ponerlas en práctica. Esta tribuna propone ampliar esta mirada hacia la pregunta por la circulación del conocimiento. En otras palabras, cómo las nuevas teorías y experiencias relativas a la planificación se difunden entre la gente y los lugares y a través de las fronteras. Debido a que la innovación está estrechamente asociada con la circulación del conocimiento, resulta difícil concebir la emergencia de la planificación urbana moderna-como disciplina y práctica distintiva- sin tal circulación del conocimiento.

En esta propuesta se examina la innovación y circulación del conocimiento en la historia de la planificación en lo que se ha considerado como región central de la planificación moderna urbana y regional, en Europa y en Estados Unidos. Finalmente, coloca algunos factores cruciales que dieron forma a estos procesos: el rol de individuos, la significación de los contextos y de factores estructurales, el rol desempeñado por los gobiernos y el lugar de un medio "reformista y técnico".

PALABRAS CLAVE | historia urbana, planificación urbana, urbanización.

ABSTRACT | Conventionally planning history is told in terms of innovations and innovators - the 'bright ideas' of planning and those who first thought of them and were able to put them into practice. This tribune aims to broaden this approach including the question of knowledge circulation. In other words how the new thinking and experience about planning spread around between people and places and across national boundaries. Considering that innovation itself is intimately associated with this knowledge circulation, it seems unlikely that modern urban planning could ever have emerged as a distinct discipline and practice without this wider circulation of knowledge.

This lecture will review innovation and knowledge circulation in planning history within what can be seen as the core or hub region of modern urban and regional planning in Europe and the United States. It will end by addressing some of the key factors which helped to shape these processes: the role of individuals, the significance of much broader contextual and structural factors, the role played by governments and the place of a "reformist and technical" milieu.

KEYWORDS | urban history, urban planning, urbanization.

E-mail: svward@brookes.ac.uk

1 Esta publicación corresponde a una ponencia dictada por el profesor Stephen Ward en octubre de 2017, en el marco del Fondecyt Regular N¹161669, a cargo de Macarena Ibarra, investigadora responsable y profesora asociada del Instituto de Estudios Urbanos y Territoriales, Pontificia Universidad Católica de Chile. Traducción del inglés por Macarena Ibarra. 


\section{Planificación urbana moderna}

La planificación urbana moderna puede ser definida como la manera de ordenar el crecimiento y el cambio urbano. En tanto disciplina, surgió cuando las ciudades crecieron a un ritmo sin precedentes, formándose grandes concentraciones de población y también de actividades económicas. Con la definición del uso del suelo urbano, los mercados se hicieron más dinámicos. La tecnología desempeñó un importante rol en la vida urbana, mientras aumentaban las presiones políticas ligadas a los nuevos emprendimientos y a las elites profesionales de las ciudades. A la vez, eran crecientes las demandas provenientes de la población misma.

En términos generales, todo ello facilitó tanto la producción económica de la ciudad como su reproducción social. A través del tiempo y en diferentes lugares, por supuesto, la relativa importancia de estas dos relaciones ha variado.

Una primera pregunta que puede plantearse es cuándo y dónde apareció esta nueva forma de planificación urbana. Sin que pueda darse una respuesta precisa, ciertamente desde mediados del siglo xIX se hizo evidente una mayor actividad destinada a regular, controlar y dar forma a las ciudades más grandes de Europa. En 1853, el Prefecto de París, Georges-Eugene Haussmann, siguiendo las indicaciones del emperador Napoleón III, puso en marcha un masivo programa de transformación de la ciudad. Se dio inicio a demoliciones, a la construcción de nuevas calles principales, de infraestructura sanitaria, de nuevos parques, y una importante reconstrucción urbana sujeta a estrictas normas de altura de las edificaciones. En 1858, el emperador austríaco Franz Joseph lanzó un proyecto con un plan de extensión para la antigua ciudad de Viena, que se ejecutaría en la gran área que la rodeaba y que anteriormente había estado ocupada por las fortificaciones de la ciudad. Otras ciudades europeas continentales estaban en lo mismo. Un plan para extender la ciudad de Barcelona preparado por el ingeniero Ildefonso Cerdà apareció el año siguiente, por mencionar un ejemplo de los planes de extensión de entonces. Muchos de ellos han sido reportados y estudiados en extenso por el historiador de la planificación sueco, Thomas Hall (1997).

Pero pese a que varios de estos planes se realizaron, la planificación urbana aún no llegaba a ser reconocible como un cuerpo de pensamiento y de práctica. Fue solo en las décadas siguientes que ello comenzó a ocurrir, a partir de iniciativas de gran peso. Más tarde, en el siglo XIX, se integraron importantes visiones e ideales de la ciudad. Estos incluyeron la idea de la ciudad lineal propuesta por el ingeniero de Madrid Arturo Soria y Mata en 1882; la noción de ciudad como una obra de arte, asociada al austriaco Camillo Sitte en 1889; y la visión de ciudad jardín propuesta por Ebenezer Howard en 1898.

También se desarrollaron nuevas metodologías y técnicas para la planificación urbana. Entre otras, normas de uso del suelo y zonificación de densidad. Se dieron también adelantos importantes en las técnicas para elaborar planes, especialmente en lo que refiere al uso de estadísticas y presentación visual. También proliferó en estas décadas la literatura relacionada con la planificación urbana, con trabajos como la Teoria General de la Urbanización (1867), de Ildefonso Cerdà; Stadt-Erweiterungen (1876), de Reinhard Baumeister; Tomorrow: A Peaceful Path to Real Reform (1898), 
de Ebenezer Howard; La Cité Jardin (1904), de Benoit-Levy; y el primer número de la revista germana-austríaca Stadtebau, en 1904.

Por esos años surgió gran parte de la terminología de la planificación urbana moderna. Muchos de los idiomas europeos acuñaron su propia etiqueta: Stadtebau en alemán desde 1889, Stadtplanung desde alrededor de 1910; Stedebouw en danés; Town Planning en inglés; City Planning en inglés americano; Urbanisme en francés; Urbanismo en español; Urbanistica en italiano y Toshi Keikaku, en japonés.

Aparecieron diversas organizaciones de redes de trabajo con interés en la planificación urbana. Algunas fueron profesionales -tal como Verband Deutsche Architekten und Ingenieur vereine o el Town Planning Institute- mientras otras tuvieron carácter más reformista, u operaron como grupos de presión o de carácter académico, tal como la Garden City Association (originalmente fundada en Gran Bretaña en 1899, pero con asociaciones similares en diferentes países en los años siguientes). Diversas conferencias y exposiciones estimularon la creatividad y el debate. Varias de ellas surgieron como parte de exposiciones internacionales más generales, pero al iniciar el siglo $\mathrm{xx}$, ya se comenzaban a especializar en asuntos de planificación urbana. Estas distintas vías que posibilitaron la circulación del conocimiento permitieron que el nuevo pensamiento que se originaba en diferentes ciudades y países fuera rápidamente cuajando y acumulándose, en un proceso que estimuló la síntesis, cambios adaptativos y ajustes diversos. Tal fue también una de las razones por las que la planificación urbana tomó forma tan rápidamente en las décadas que precedieron a la Gran Guerra.

Así, se puede reconocer que esos años fueron conducentes a la circulación del conocimiento. Y si hoy estamos familiarizados con el término 'globalización', entonces, cuando la planificación urbana moderna realmente surgió, también se estaba frente a una era global, incluso más que la actual.

\section{La influencia europea y estadounidense: contextos e individuos}

Durante el siglo XIX, hubo significativos avances en las comunicaciones. Prosperó la prensa escrita y se desarrolló rápidamente el sistema de transporte, en especial el ferrocarril y la navegación a vapor. No solo se trasladaban personas, el correo y la prensa; también a los largo de sus rutas las líneas del telégrafo marcaron los inicios de las telecomunicaciones.

Pese a guerras continentales de corta duración, el largo siglo xix tuvo un periodo de relativa paz, especialmente entre 1815 y 1914. Es más, a partir de la década de 1860 y hasta 1914, gran parte de Europa pasó a ser zona libre de pasaportes y visas.

Entretanto, a fines del siglo XIX, se experimentó una expansión marítima masiva de rutas comerciales y de imperios de ultramar de las grandes potencias europeas. Esto reforzó o creó nuevas esferas en las que el pensamiento europeo se diseminó. En un contexto imperial, ello ocurrió usualmente como una imposición que no habría sido posible en la madre patria... o tierra paterna. Las posiciones coloniales no tuvieron la posibilidad de elegir el origen de sus ideas de planificación. En la Francia imperial, ello ocurrió desde París; en la inglesa, desde Londres; en la Alemania imperial, desde Berlín, entre otros. En países con algún nivel de autodeterminación 
local (por ejemplo, en los dominios británicos), hubo más posibilidad de elección; así ocurrió en los casos de Canadá y Australia, que comenzaron a abrirse a ideas de los Estados Unidos. Pero esto no fue lo usual.

Algunos países, como España, experimentaron una contracción de su alcance imperial, con la consiguiente reducción del impacto del pensamiento español sobre desarrollo urbano. En sus antiguas colonias latinoamericanas se generó una mayor atención hacia las fuerzas mundiales en ascenso en esos días, como Francia (especialmente desde antes de 1914), pero también Gran Bretaña, Alemania y los Estados Unidos.

Al momento de la Primera Guerra Mundial, era ciertamente evidente que las ideas de planificación urbana moderna habían avanzado más allá de su punto de origen. Así, en las zonas más periféricas de Europa se estaban adoptando e implementando distintas concepciones de planificación. No obstante, el estallido de la guerra interrumpió tal internacionalización y los efectos de varios cambios fueron decreciendo en los ańos veinte, pese a que algunos de ellos permanecieron, tales como los relativos a pasaportes y visas. Las comunicaciones internacionales, fortalecidas con las rutas aéreas, fueron utilizadas también desde las décadas de 1920 y 1930 por algunos planificadores urbanos.

Sin embargo, el mayor impacto provino de los extraordinarios cambios políticos que generó la guerra. El más importante fue la Revolución Rusa y la creación de la Unión Soviética. En Europa del Este, los antiguos imperios continentales rusos, austriacos y otomanos derivaron en una serie de nuevos estados independientes. A partir de la década de 1920, la Unión Soviética pasó a ser una nueva fuente de pensamiento y de práctica de la planificación, atrayendo el interés occidental en los ańos treinta. En Occidente también se generaron nuevas ideas como parte del movimiento internacional de arquitectura moderna, asociado a la organización del CiAm (Congreso Internacional de Arquitectura Moderna) en 1928.

Hubo otros cambios notables relacionados con la agenda de la planificación en la Europa occidental y central en el periodo de entreguerras. Los aspectos sociales de la planificación urbana adquirieron mayor peso, en especial los relacionados con la vivienda social.

Otra dimensión importante de la planificación urbana después de 1919 fue el rol que esta disciplina desempeñó en cuanto a forjar una mayor identidad en los nuevos estados nacionales de Europa del Este. Algunos ejemplos de ello fueron el famoso plan de Eliel Saarinen para Helsinki, capital de la recién independiente Finlandia, durante la posguerra; el plan para la nueva capital del estado de Turquía, Ankara, preparado a fines de los ańos veinte por Herman Jansen como reemplazo de la vieja capital otomana de Estambul. Ambos casos expresan la confianza de las nuevas naciones tras independizarse de antiguos imperios.

También significativa del periodo de entreguerras fue la planificación para vehículos motorizados. Alemania y Estados Unidos surgieron como líderes en este campo. Tal fue el caso del famoso plan para el primer barrio de Radburn, una ciudad jardín en New Jersey, cuya principal característica fue el alto grado de separación entre los vehículos motorizados y la circulación peatonal. Contaba con redes de rutas peatonales completamente separadas de las calles, con parques interiores 
e incluso con los primeros pasos peatonales bajo nivel. Esta iniciativa marcó una puesta al día de la ciudad jardín en la era motorizada.

Hacia la década de 1930, Estados Unidos era pionero en la planificación de autopistas y Nueva York inauguraba las primeras al final de la década. Probablemente no se trata del aporte más satisfactorio de Norteamérica a la planificación, pero, sin duda, es el más importante. Sin embargo, fue en Alemania, durante el periodo nazi, que aparecieron las carreteras interurbanas de alta velocidad. Pese a que el uso de automóviles era mucho más bajo que en Estados Unidos y varios otros países, el gobierno de Hitler construyó una red nacional con esas vías, las que fueron ampliamente admiradas y estudiadas en diversos lugares.

No obstante, fue otro estado totalitario, la Unión Soviética, el que sentó las bases de un notable nuevo campo de la planificación, cuyo foco de interés era el desarrollo regional y económico. El primer Plan Quinquenal de 1928 propugnaba una industrialización a gran escala y la correspondiente urbanización. Dicho plan y los siguientes atrajeron interés internacional. Proyectos emblemáticos fueron Magnitogorsk, la nueva ciudad de hierro y acero, y los masivos proyectos en el río Volga.

La gran pregunta sobre si los países democráticos podrían planificar tan efectivamente como parecían hacerlo la Unión Soviética, la Alemania nazi y otros estados totalitarios, adquirió gran relevancia en la década de 1930. En 1933, el nuevo presidente de Estados Unidos, Franklin Delano Roosevelt, lanzó su política New Deal, que ofrecía una robusta alternativa democrática.

Los planificadores urbanos y regionales de otros países tuvieron especial interés en los programas urbanos de cinturones verdes y en el masivo proyecto del valle de Tennessee, ciudad con un cinturón verde cerca de Washington D.C., que pasó a ser un modelo de desarrollo comunitario y de nueva planificación urbana. Este proyecto incorporó la recuperación de los suelos y del paisaje, la generación hidroeléctrica, nuevos edificios para vivienda y proyectos de desarrollo turístico.

Entretanto, los medios de circulación de conocimiento sobre planificación a nivel internacional que habían sido interrumpidos con la Primera Guerra se restablecían. Nunca volvieron a ser tan libres como lo habían sido hasta antes de 1914. Pero la creciente eficiencia del transporte internacional y de las comunicaciones, incluyendo los inicios del transporte aéreo al menos para trayectos cortos, compensó parcialmente sus restricciones. También se fortalecieron las organizaciones de redes internacionales.

Con el estallido de la Segunda Guerra Mundial en 1939, varias de las conexiones señaladas se vieron interrumpidas. Planificadores alemanes habían visitado Gran Bretaña pocos días antes de que la guerra estallara, pero un grupo de arquitectos británicos fue detenido en la Unión Soviética cuando recién había anunciado su alianza con Alemania. Más allá de todo ello, el principal efecto de la guerra fue la destrucción -especialmente a través del bombardeo aéreo- que devastó muchas ciudades. Varias ciudades británicas se vieron muy afectadas, aunque menos que otras del continente, como ocurrió en Alemania, Polonia y la parte occidental de la Unión Soviética.

Lo anterior explica que la destrucción de la posguerra fuera el tema que dominó a Europa después de 1945, por al menos una década. Las respuestas frente a este 
asunto tuvieron marcadas diferencias. En un extremo, la aproximación fue histórica y prevaleció en Europa, pero de manera más completa en Polonia, especialmente en Varsovia. Incluso en este caso, usualmente se implementó una cierta modernización (por ejemplo, un túnel para el tránsito automotor y tranvías bajo la antigua ciudad, esperadamente reconstruida). En el Este comunista fue más común un enfoque etiquetado de socialismo realista, como lo ejemplificara Stalinallee en Berlín. Se trataba de un bulevar monumental enmarcado por construcciones de varios pisos de fachada continua, con imponentes edificios o torres en intersecciones o vías de acceso cruciales. Los edificios eran esencialmente modernos, pero su estilo arquitectónico evocaba temas germánicos, a la vez que se adecuaba a la ideología socialista. Por contraste, las ciudades occidentales fueron de carácter más modernista en su arquitectura y planificación, creando a menudo nuevos trazados de calles y patrones constructivos.

Otro tema que dominó en la Europa de la posguerra, y en menor medida en Estados Unidos, fue la planificación de nuevas ciudades. Los más tempranos ejemplos occidentales se dieron en Suecia e Inglaterra, con Vallingby, la famosa nueva ciudad al oeste de Estocolmo, y Stevenage, respectivamente. Vallingby estaba vinculada directamente con Estocolmo; por tanto, no era una ciudad totalmente autosuficiente. Stevenage, más distante de Londres, por contraste, fue planeada para autosustentarse. Como fuere, las nuevas ciudades suecas y británicas gozaron de características comunes, lo que incluía un apego a ciertos principios de barrio para la vivienda y la provisión de servicios locales.

$\mathrm{Al}$ otro lado de la cortina de hierro también se construyeron nuevas ciudades, como lo fueran Magnitogorsk o Nowa Huta (la nueva ciudad que colindó con el centro histórico de Cracovia), las cuales estuvieron comúnmente asociadas al desarrollo de la industria pesada. Nowa Huta, construida para alojar a los obreros de la industria acerera, siguió los principios del realismo socialista, con calles monumentales, alineadas con edificios imponentes. Una ciudad más tardía se aprecia en el París metropolitano, con Cergy-Pontoise, a principios de los años setenta. Tal como las nuevas ciudades suecas, esta fue una ciudad satélite, muy vinculada al mercado laboral metropolitano y, por tanto, menos autosuficiente que sus pares británicos.

Hacia los años sesenta, la agenda de la planificación de las grandes ciudades había dejado atrás su foco en la reconstrucción de la posguerra y avanzaba hacia la modernización de ciudades, especialmente en relación con la planificación de vehículos motorizados.

En Europa se dio una gran dependencia de las experiencias de ciudades de Estados Unidos. Además de la pericia norteamericana en la planificación de autopistas, sus nuevas formas de consumo se replicaron ampliamente. El Penn Center en Filadelfia, planeado a inicios de 1950, pasó a ser un modelo que combinaba el desarrollo de un centro comercial de varios pisos con acceso de transportes en el corazón de las ciudades. Se estudiaron las diversas maneras de incorporar autopistas en la forma urbana. Las ciudades europeas comenzaron a imitar varias de estas tendencias, iniciando amplios procesos de remodelación urbana en los años sesenta.

Hubo también otro enfoque. En Alemania, ciertos centros históricos de inicios de los años cincuenta se habían hecho peatonales en el marco de los trazados existentes, 
con exclusión de tráfico de estas zonas. Esto implicó una menor reurbanización, pero sí algunas restricciones al uso de vehículos motorizados, particularmente automóviles. Tal aproximación no generó de inmediato la enorme atracción de aquellas ciudades completamente modernizadas donde no había zonas vedadas para el uso del automóvil.

El periodo de boom económico de la posguerra fue también importante para la planificación en el resto del mundo. Los antiguos imperios coloniales europeos llegaban a su fin. Lo que siguió fue un fértil terreno para los planificadores. Al igual que los nuevos estados nacionales surgidos después de la Primera Guerra Mundial, los países poscoloniales recientemente independizados vieron la planificación como una manera de establecer sus nuevas identidades como países y de encarar sus necesidades más urgentes. Y ello con una importante diferencia entre ambos grupos: varias de estas nuevas naciones buscaron ayuda para su desarrollo y asistencia técnica en países con mayores recursos, pero esta asistencia no provino ya de los antiguos poderes coloniales. El mundo poscolonial pasó así a ser un importante escenario de acción para los planificadores de muchos otros países, usualmente como parte de paquetes de ayuda de sus propios gobiernos o porque habían sido recomendados por la oficina o agencia de planificación de las Naciones Unidas (precursora de la actual Hábitat).

Desde fines de los setenta se dieron cambios decisivos en la economía mundial, y el capital se hizo cada vez más móvil. Antiguas regiones industriales, tales como Europa y el noreste de Estados Unidos, comenzaron a experimentar serias desinversiones con el cierre de fábricas. El apoyo político en todas partes se distanció del tipo de planificación y de los grandes proyectos gubernamentales que habían estado en boga en los ańos sesenta. La agenda social de la planificación se debilitó. Gradualmente, la agenda política se hizo más dependiente del mercado. A fines de los ochenta e inicios de los noventa, incluso el comunismo iba colapsando o cambiando su naturaleza para adoptar una política económica más cercana al mercado neoliberal.

En Europa y en Estados Unidos, surgieron nuevas aproximaciones a la planificación. El marketing de ciudades se hizo común, siguiendo la pauta de la campaña 'I love NY', inaugurada en 1977 en Nueva York. Otros países rápidamente siguieron esa línea. La idea de Enterprise Zones (zonas empresariales) también apareció a inicios de los ochenta. Fue un intento por capturar algo del modelo de crecimiento que parecía tan exitoso en las ciudades conocidas como "tigres asiáticos". En ellas los gobiernos habían definido zonas con diversas rebajas de impuestos y más flexibilidad de planificación, dando mayor libertad al crecimiento y desarrollo de negocios. Primero apareció en Gran Bretaña en 1980, y luego en Estado Unidos. Con el tiempo, esta idea se difundió a otros países, por ejemplo a Francia en 1996.

Pero quizá el tipo de desarrollo más característico asociado al neoliberalismo fue la remodelación de antiguas áreas portuarias. El proyecto de Baltimore fue uno de los primeros en ser ampliamente reconocido, desde fines de 1970, y a su vez fue extensamente emulado. Introdujo actividades turísticas y de otros tipos. Sin embargo, el más importante fue el proyecto de remodelación de los muelles de Londres (London Docklands Regeneration Project), donde también se dieron elementos del enfoque de 
Baltimore, pero igualmente un desarrollo comercial a gran escala (especialmente en Canary Wharf), acompańado de desarrollo residencial.

En los noventa, sin embargo, el liberalismo aparecía crecientemente morigerado, con un creciente énfasis en la sustentabilidad ambiental y en la idea de hacer de las ciudades lugares más "vivibles". Europa continental produjo importantes ejemplos. Stroget, la principal zona peatonal del centro de Copenhague, desde los setenta es un recurrido lugar de vida al aire libre, centro de muchos eventos sociales formales e informales. Otra capital escandinava, Estocolmo, desde los noventa cuenta con un importante distrito ecológico en una antigua zona industrial en Hammarby Sjostad, que incluye los últimos avances en generación energética, manejo de basuras, transportes, entre otros. Alemania también aparece como un importante referente. En los ańos noventa, en la International Building Exhibition en Emscher Park, en el área metropolitana del Ruhr, fue anfitrión de proyectos individuales. Central fue el reclamo por el fuertemente contaminado río del valle Emscher. Pero quizá un ejemplo más emblemático fue Freiburg, donde el distrito Vauban pasó a ser el primer caso de planificación ecológica para una mayor sustentabilidad en el país.

\section{Reflexiones finales}

Esta sucinta revisión de la historia de la planificación desde una perspectiva europea da cuenta de una serie de innovaciones que tuvieron lugar durante fines del siglo XIX e inicios del xx en diversos países de la Europa occidental y central y, posteriormente, en Estados Unidos. Su impacto tuvo mayor alcance gracias a los efectivos medios de circulación de conocimiento que rápidamente los aglutinaron, especialmente en Europa. Estas innovaciones ocurrieron principalmente en los países más industrializados y urbanizados del momento y/o los que tenían una red imperial o semiimperial.

Las innovaciones subsiguientes fueron más bien refinamientos lineales de lo ya establecido o, más bien, nuevas síntesis de ideas puestas en conjunto; en algunos casos, nuevos ciclos creativos que surgieron como respuestas a las nuevas circunstancias.

Entonces, ¿qué podemos decir acerca de los nuevos factores que han moldeado este proceso de innovación y de circulación de conocimiento?

La historia de la planificación es generalmente protagonizada por individuos, tales como Idelfonso Cerdà, Camillo Sitte, Ebenezer Howard y Le Corbusier, entre otros. Pero, ¿cuán significativos son los individuos en la historia? Como se ha visto, la innovación y sus contribuciones no ocurrieron aisladamente. El hecho de que los tempranos esfuerzos de Cerdà en 1867 en cuanto a proponer una teoría general de la planificación (o de 'urbanización', como la designó) fuesen casi completamente ignorados en otros países, plantea una pregunta central sobre la real importancia de las figuras individuales.

La respuesta puede hallarse parcialmente al otro lado del espectro, entre aquellos factores contextuales y estructurales de más largo alcance. Ciertamente, la planificación surgió en los países de mayor poder económico y geopolítico de fines del siglo diecinueve e inicios del veinte. El hecho de que por ese entonces Espańa fuera 
un país cuyo poder estaba en declive, podría explicar por qué Cerdà fue pasado por alto. Aunque tal vez esta no fue la única razón, pues posteriormente las ideas de ciudad lineal de Arturo Soria no tuvieron el mismo destino.

Pero, ciertamente, la historia de la planificación está entretejida con los cambios políticos del poder global, como lo demuestra - por ejemplo- el auge de la Unión Soviética con una nueva idea de planificación. Sin embargo, la historia de la planificación también hace ver que países más pequeños y menos poderosos, tales como los escandinavos y Holanda, han desempeñado importantes roles; hoy también podría considerarse a Singapur. Tampoco se puede perder de vista la importancia que el idioma y las comunicaciones tienen en estas materias: los tres más importantes fueron el inglés, el francés y el alemán, mientras el ruso no logró influenciar más allá de sus fronteras.

En relación con el rol de los gobiernos, se puede señalar que no destacan por ser agencias creativas, sino más bien por poner en marcha ciertas tareas rutinarias, aunque ciertamente existen circunstancias donde los gobiernos pueden liderar en innovación o en introducir un nuevo pensamiento proveniente de fuentes externas. Por ejemplo, trastornos como una guerra pueden estimular innovación, según ocurrió en diversos países europeos después de la Segunda Guerra Mundial. También puede ser creativo el periodo anterior al conformación plena del Estado. Se viene a la mente el ejemplo de la Unión Soviética en los primeros años tras 1917, pero también el caso de los muchos estados nacionales surgidos desde la Primera Guerra Mundial.

Los gobiernos también pueden decidir incentivar un pensamiento renovado. Por ejemplo, en Gran Bretańa y Francia, en la década de 1960, específicamente convocaron a nuevas ideas. En Gran Bretaña, la pregunta acerca de cómo planificar las áreas urbanas para el tráfico motorizado fue central. En Francia, fue acerca de cómo planificar el área metropolitana de París. En el marco de este mismo espíritu es que hay que entender las International Building Exhibitions (IBA) en Alemania, tal como el caso de Emscher Park.

Otro factor importante que incide en la planificación urbana es lo que podría llamarse el medio reformista y técnico, referido a organizaciones no gubernamentales, redes e iniciativas que han aportado y dado forma a la planificación. Estos actores han enmarcado el debate y la evaluación de innovaciones, han tenido un rol central en la circulación del conocimiento y, sin dudas, han sido fundamentales en la síntesis, adaptación y ajustes del conocimiento recibido. También han promovido y publicado importante literatura sobre la planificación, desarrollado conferencias, charlas, exhibiciones y encuentros, entre otros eventos. Se trata de mentores cruciales para el desarrollo de la planificación.

Los individuos pueden desempeñar un valioso rol creativo. Sin embargo, el alcance de sus ideas -esto es, hasta dónde tienen algún efecto- depende del marco en el que se gestan o en el que se reciben. Por lo general, lo que tiene lugar en países grandes y poderosos tiene mayores posibilidades de ser tomado en cuenta. No obstante, es mi parecer que tanto los gobiernos como ciertas entidades reformadoras y técnicas pueden tener funciones importantes, en parte autónomas, en la posibilidad de relacionar a los individuos con esas fuerzas mayores. 


\section{Referencias bibliográficas}

Baumeister, R. (1876). Stadt-Erweiterungen. En Technischer, baupolizeilicher und wirthschaftlicher Beziehung. Berlin: Ernst \& Korn.

Benoit-Levy, G. (1904). La Cité Jardin. Paris: Henri Jouve.

Cerdà, I. (1867). Teoría general de la urbanización y aplicación de sus principios y doctrinas a la Reforma y Ensanche de Barcelona. Madrid: Imprenta Española.

Hall, T. (1997). Planning Europe's capital cities: Aspects of nineteenth-century urban development. London: Routledge.

Howard, E. (1898). To-morrow: a peaceful path to real reform. London: Swan Sonnenschein. 